\title{
La Didáctica de las Ciencias Experimentales en el Grado en Maestro/a de Educación Primaria
}

Jaime Nácher-Mestre nacher@uji.es

Lidón Monferrer Sales lidon.monferrer@uji.es 
Los estudios universitarios se consideran una etapa educativa avanzada que requiere una implicación relevante del estudiante y del profesorado. Concretamente en el Grado en Maestro/a de Educación Primaria se proporcionan conocimientos, actitudes y habilidades para que los futuros maestros/as sean autónomos y eficaces en la escuela. En general, aquellos cursos y/o asignaturas con base teórica 0 no directamente aplicada generan apatía en el estudiante y en este sentido, la Didáctica de las Ciencias Experimentales es comúnmente percibida como demasiado formal, abstracta y su puesta en práctica no despierta interés en el alumnado.

A finales del curso 2013/14 se realizó un estudio sobre el alumnado de 20 curso para cuantificar el interés por la Didáctica de las Ciencias y su capacidad para enseñar y promocionar las Ciencias en la escuela. Los resultados mostraron que los alumnos carecen de cierto perfil científico pues llevan muchos años sin cursar Ciencias. De esta forma resulta poco probable su futura implicación en Ciencia y su promoción en la escuela. El interés por la Ciencia desde el inicio hasta el final del curso mejoraba sensiblemente pero aun así, la valoración final es inferior a la esperada. Por último, conviene destacar que aproximadamente el 30\% del alumnado encuestado no se siente capacitado para enseñar Ciencias en la escuela.

Para mejorar la percepción de la Ciencia se proponen una serie de propuestas entre las que destacan una mayor interacción profesor/alumno para desarrollar actividades docentes en el aula escolar. Paralelamente se plantea que el docente universitario se implique en cursos de formación del profesorado para mejorar la transferencia de conocimiento. También se tratará de mejorar la interacción Ciencia-Tecnología-Sociedad con el fin de promocionar la Ciencia en la vida real y que esta situación sea interpretable por los estudiantes. Por último, promocionar la investigación en Didáctica de las Ciencias y aportar una visión crítica serían también pilares básicos sobre los que mejorar la docencia y despertar el interés de la Ciencia en los alumnos.

Palabras clave: Ciencias experimentales, Didáctica, Grado en Educación Primaria, Maestro/a, Escuela, Ciencia-Tecnología-Sociedad, Investigación.

\section{Introducción}

La importancia de adquirir conocimiento relacionado con la Ciencia debería estar fuera de toda discrepancia. Pensar que la sociedad puede mantenerse alejada de disciplinas que explican procesos naturales que nos envuelven y rodean es totalmente erróneo. La sociedad se ha desarrollado gracias a disciplinas científicas como la química, física, biología, farmacia, etc. y sin ellas, la sociedad carecería de avance, de evolución (FECYT, 2013:17). 
Estas premisas que deberían ser palpables en la sociedad no lo son tanto en diferentes niveles de la educación contemporánea. A pesar de que datos públicos reflejan que el interés por la Ciencia y la Tecnología crece un 19\% desde 2010 (FECYT, 2012:3), otras tendencias apuntan el descenso de alumnos en estudios de Ciencia y Tecnología en Educación Secundaria y Bachillerato además de observarse que el número de estudiantes en los primeros cursos universitarios de las ramas experimentales y técnicas ha descendido en los últimos diez años (FECYT, 2013:30; Hernández Armenteros, 2010).

Existen diferentes planteamientos sobre el deterioro de las actitudes relacionadas con la Ciencia a medida que crece la edad de los estudiantes. Los alumnos manifiestan una percepción de las carreras científicas como aquellas que contienen clases poco atractivas y de elevada dificultad. Paralelamente, entre los jóvenes también hay bajas perspectivas laborales y de salario (FECYT, 2013:31).

El alumnado de Grado en Maestro/a de Educación Primaria no representa una excepción al rechazo por las Ciencias. En las asignaturas de Didáctica de las Ciencias Experimentales muchos alumnos presentan carencias conceptuales que dificultan la posibilidad de capacitarse como futuros docentes y primeros promotores de la Ciencia en la escuela. El perfil de estudiante matriculado en el Grado en Maestro/a de Educación Primaria es muy diverso y se pueden encontrar alumnos de diferentes edades, procedencias, estudios previos, expectativas de presente y de futuro, etc. (Camina Durántez y Salvador Pérez, 2007). Todo este compendio de características hacen que la actividad docente universitaria sea compleja. El docente universitario deberá desarrollar capacidades para poder atender tanta variedad de necesidades dentro de un modelo de enseñanza con grupos de alrededor de 90 estudiantes por clase.

A nivel escolar, muchos maestros/as carecen de un conocimiento básico de Ciencia elemental apropiada para los niños de esa edad. Esta situación es una de las mayores dificultades para mejorar la docencia en Ciencia en Educación Primaria (Cortés Gracia, 2012; Pujol, 2008).

La actividad del docente de Didáctica de las Ciencias Experimentales es vital para trasladar a los futuros maestros/as propuestas para contrarrestar la ausencia de un gran interés y promoción de la Ciencia en Educación Primaria. El docente universitario tiene que saber diseñar y proporcionar una construcción colaborativa que de respuesta a tantos interrogantes que le aparecen al estudiante de Grado (Cañal, 2007). Es urgente cambiar el modelo conceptual y teórico no directamente aplicado por una modalidad en la que el docente universitario deberá saber enseñar Ciencias, enseñar a integrarlas en el grupo de escolares y que éstas sean dinámicas en los centros escolares (Pujol, 2008). Sólo así, se completará el deseo de saber y de comprender que tienen los maestros/as acerca de cómo enseñar Ciencia a los escolares.

En el presente trabajo se muestran los resultados procedentes de un estudio sobre la percepción de la Didáctica de las Ciencias Experimentales en alumnos/as de Grado en Maestro/a de Educación Primaria. A partir de 
su discusión se realizan propuestas a llevar a cabo con el fin de mejorar la percepción de la Ciencia en los alumnos, futuros maestros/as y primeros promotores de la Ciencia a nivel educativo.

\section{Objetivos}

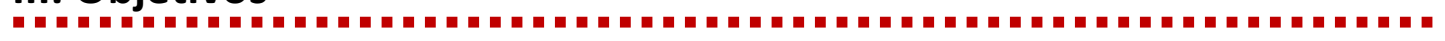

Los objetivos principales planteados en el presente trabajo son cuantificar el interés por la Didáctica de las Ciencias Experimentales y la predisposición para enseñar Ciencias de los alumnos del Grado en Maestro/a de Educación Primaria. Asímismo, se plantean propuestas que ayuden a mejorar la percepción y promoción de la Ciencia en los futuros maestros/as.

\section{Material y métodos}

El estudio sobre la percepción actual de la Didáctica de las Ciencias Experimentales se ha llevado a cabo en alumnos de 20 curso que acababan de cursar las asignaturas de Didáctica de la Física y Química y Didáctica de las Ciencias Naturales, del Grado en Maestro/a de Educación Primaria de la Universidad Jaume I (Castellón, España). El estudio se llevó a cabo mediante la realización de un cuestionario anónimo de respuesta corta y directa (Anexo I).

Se trata de un cuestionario en el que destacan, entre otras, preguntas sobre la modalidad de ingreso en la Universidad, cúal ha sido la primera opción de carrera universitaria, cuantos años hace que se cursaron asignaturas de Ciencias por última vez y finalmente, si el alumno/a de grado, futuro maestro/a, fomentaría el conocimiento e interés científico y si se siente capacitado para ello. El cuestionario se llevó a cabo al final del curso 2013/14, concretamente el 26 de mayo de 2014.

\section{Resultados y discusión}

5.1 Percepción actual de los estudiantes universitarios hacia la Didáctica de las Ciencias Experimentales

El presente trabajo no ha llevado a resultados muy satisfactorios desde el punto de vista de la promoción de la Ciencia. Los resultados obtenidos están en concordancia con la tendencia observada en años anteriores por Vázquez y Manassero (2008). Los resultados del estudio realizado pueden consultarse en la Tabla 1. 
Tabla 1. Cuestionario sobre la percepción actual de los alumnos sobre las Ciencias Experimentales y su Didáctica. Resultados.

Didáctica de las Ciencias Experimentales

Promoción de la Ciencia en la Escuela

\begin{tabular}{|c|c|}
\hline & $(n=70)$ \\
\hline Edad & 20,71 \\
\hline Lugar de nacimiento & - \\
\hline № veces matriculado/a & 1,03 \\
\hline 1. ¿En qué localidad has cursado el Bachillerato o Formación Profesional? & - \\
\hline 2. ¿Has hecho la Selectividad? SI/NO, ¿Qué nota has obtenido sobre 10 ? & $84 \%(\mathrm{SI}) ; 7,00$ \\
\hline 3. ¿Fue el Grado en Maestro/a de Educación Primaria tu primera opción? SI/NO & $24,25 \%(\mathrm{NO})$ \\
\hline \multicolumn{2}{|l|}{ 4. ¿Por qué motivo has decidido estudiar esta carrera? } \\
\hline 5. ¿Cuántos años hace que estudiaste alguna asignatura de Ciencias? & 4,12 \\
\hline 6. ¿Qué interés tenía por la Didáctica en Ciencias al inicio de esta asignatura? Pon nota & 5,94 \\
\hline 7. ¿Qué interés tienes por la Didáctica en Ciencias al finalizar esta asignatura? Pon nota & 6,58 \\
\hline 8. ¿Fomentarías el conocimiento e interés científico cuando seas maestro/educador? & $98,48(\mathrm{SI})$ \\
\hline 9. ¿Te sientes capacitado para fomentar la Ciencia en los escolares? SI/NO & 28,79 (NO) \\
\hline \multicolumn{2}{|l|}{ 10. Introduce cualquier comentario anónimo sobre cómo incentivar el interés por la } \\
\hline Ciencia y su didáctica en los futuros maestros: & \\
\hline
\end{tabular}

Las preguntas 1, 4 y 10 (Tabla 1 ) tienen una gran variabilidad de respuestas y no han sido incluidas en la tabla. Cabe destacar que las respuestas van dirigidas en la misma línea que proponían Camina Durántez y Salvador Pérez (2007) sobre un perfil de estudiante muy heterogéneo, que puede llegar a comprometer la labor del docente universitario. Se trata de estudiantes que proceden de localidades geográficas diferentes, principalmente a nivel provincial y autonómico. Las principales motivaciones apuntadas por los alumnos/as para matricularse en el Grado han sido su aprecio por los niños pequeños y vocación docente. Respecto a la pregunta 10 (Tabla 1), aproximadamente un 65\% del alumno encuestado ha incluido algún comentario que hace que esta pregunta haya sido muy positiva pues, en general, se remarcan aspectos que no han gustado al alumnado y que emplazan a posibles mejoras. Cabe destacar que los comentarios van relacionados con la demanda de mayor carga práctica experimental que pueda ser aplicable posteriormente en la escuela. También se demanda eliminar las clases magistrales basadas en aprendizaje conceptual proponiendo el uso de metodologías atractivas para la Didáctica de la Ciencia. 
Los resultados procedentes de las preguntas con respuesta numérica se discuten a continuación. En primer lugar, la edad de comienzo del 20 curso de la carrera se esperaba que fuera ligeramente superior a 19 años. Esta edad es la correspondiente a estudiantes en condiciones normales (procedentes de Bachillerato y sin haber perdido ningún curso). La media de edad de los estudiantes encuestados fue sensiblemente superior a lo esperado (20.71 años). Se han revisado resultados paralelos a este estudio, realizados en la misma Universidad (información no publicada), que muestran que esta cifra podría verse incrementada considerando los cursos de la tarde en el que están matriculados muchos alumnos que accedieron a la Universidad mediante pruebas de acceso a mayores de 25 años. Ésta también puede ser la causa de que un $16 \%$ de los alumnos encuestados no accedieran a la Universidad mediante Selectividad frente al $84 \%$ que sí que accedieron mediante Selectividad (nota media de acceso de 7 sobre 10, pregunta 2, Tabla 1). Esta dispersión de edades entre el alumnado también ha sido reflejada previamente en otros estudios que muestran gran variedad de experiencias y motivaciones en el alumnado previas al acceso a los estudios universitarios (Camina Durántez y Salvador Pérez, 2007).

Más de un $75 \%$ de los alumnos encuestados seleccionaron el Grado en Maestro/a de Primaria como primera opción (pregunta 3, Tabla 1). Como se ha comentado anteriormente, hay un gran porcentaje de alumnos que presentan pasión por los niños y además sienten la vocación docente. Igualmente, esta carrera puede ser una prioridad en muchos estudiantes debido a la prosperidad laboral que han tenido promociones anteriores durante años. También se considera una carrera concebida como "asequible" para personas jóvenes y más adultas que presentan una mayor ocupación y/o responsabilidad laboral o familar.

Como mínimo, nuestros estudiantes llevan una media de 4 años sin estudiar asignaturas de Ciencias (pregunta 5, Tabla 1). Es uno de los peores resultados que podíamos esperar. Paralelamente, el perfil de estudiantes que han estudiado Ciencias en Bachillerato es muy bajo. Únicamente un $10 \%$ del alumnado encuestado proviene de Bachillerato con asignaturas de Ciencias. Ante esta situación, es difícil esperar que esta promoción de futuros maestros/as transmitan interés por las Ciencias si ellos actualmente carecen. Este resultado también implica un problema en el aula pues no es fácil solventar errores o falta de conocimiento de nivel de Secundaria en 20 curso de grado universitario y más cuando los esfuerzos se deberían dedicar a la Didáctica de las Ciencias.

A pesar de este hecho, en general, tras cursar las asignaturas de Didáctica de las Ciencias Experimentales, los alumnos/as muestran creciente interés por la Ciencia. Este interés se refleja en un crecimiento cercano al $6 \%$ desde el inicio de las asignaturas hasta el final de las mismas (pregunta 6 y 7, Tabla 1). Aún así, el alumnado encuestado propone una valoración media que no llega al notable (6.58). La VI Encuesta de Percepción de la Ciencia y Tecnología (FECYT, 2012) ya destacó el aumento 
del interés por la Ciencia y la Tecnología entre los jóvenes aunque perciben el nivel de educación científica recibido como bajo o muy bajo.

Por último, las cuestiones sobre la disponibilidad de los alumnos a fomentar las Ciencias (pregunta 8 y 9, Tabla 1) muestran resultados sobre un mayoritario acuerdo en el fomento de conocimientos e interés por las Ciencias pero cerca de un $28 \%$ de los alumnos no se considera capacitado para llevarlo a cabo en Educación Primaria. Parece evidente que es necesario un esfuerzo en formar mejor a los universitarios, futuros maestros, para que sean capaces de aplicar un mayor contenido de Ciencias en Educación Primaria y más teniendo en cuenta la dificultad que entraña el perfil del alumnado del Grado en Maestro/a de Educación Primaria.

Resultados obtenidos en el presente estudio también han sido observados en otros centros europeos. Schreiner y Sjøberg (2004) y Sjøberg (2005) muestran una amplia colección de datos sobre la problemática de la Didáctica de la Ciencia y la Tecnología. A pesar de la gran importancia que tienen estas disciplinas en la sociedad moderna, mucha gente joven pierde el interés el interés por la Ciencia y Tecnología en la escuela y estudios posteriores (Sjøberg y Schreiner, 2010).

\section{2. Propuestas aplicadas para mejorar la percepción de las Ciencias Experimentales}

Los planes futuros son muy amplios, también las posibilidades y retos a abordar para mejorar la Didáctica de las Ciencias en el Grado en Maestro/a de Educación Primaria dentro de esta Universidad. Proponemos a continuación líneas muy generales que se tendrán en consideracíón para tratar de solventar las conclusiones más desfavorables que nos hemos encontrado.

Un primer planteamiento sería, a partir de situaciones reales, incentivar a los estudiantes para proponer respuestas a cuestiones que el docente plantee relacionadas con la Ciencia en la vida real. El docente deberá explicar el fundamento teórico y de esta manera el alumnado adquiere una visión más completa y razonada. Seguidamente los alumnos universitarios, junto al profesor, tendrán que diseñar actividades que estén destinadas a escolares y así capacitarlos a entender la situación real con explicación de base científica. Tras la evaluación, discusión y aprobación de los compañeros y del profesor, estas actividades se podrían aplicar en futuras prácticas docentes en la escuela. Un buen momento para su realización sería en cursos siguientes, cuando los alumnos estén cursando cualquier asignatura de Practicum o bien, durante el último año como parte del trabajo final de Grado.

Paralelamente, se plantean una serie de propuestas sobre las que trabajar. Hay que destacar que todas ellas están fuertemente vinculadas entre sí y la aplicación de una junto a las demás mejora la efectividad conjunta. La primera consiste en la participación de los docentes universitarios de Didáctica de las Ciencias en cursos de formación del profesorado que permitan mejorar la transferencia de la Didáctica de las 
Ciencias y hacer más visible su aplicación en la escuela. En segundo lugar, se tratará de mejorar la interacción Ciencia-Tecnología-Sociedad (CTS) con el fin de promocionar la relación de la Ciencia con la vida real y así ser fácilmente interpretable por los estudiantes. Parece evidente incluir esta aplicación en el aula para mejorar el aprendizaje pero resulta complejo llevarla a la práctica pues influyen muchos factores en la consecución de actitudes positivas hacia la relación CTS y educar en Ciencia (VázquezAlonso, 2006).

Finalmente se anima al profesorado universitario a desarrollar y ampliar su faceta investigadora. Es muy importante la redacción y participación en proyectos de investigación que incentiven la investigación y desarrollo de la Didáctica de las Ciencias. También la redacción e interpretación de resultados como el del presente trabajo así como su divulgación en artículos de investigación o presentación en jornadas, reuniones y/o congresos. Promocionar la investigación en Didáctica de las Ciencias Experimentales y aportar una visión crítica serían pilares básicos sobre los que mejorar la docencia y despertar el interés de la Ciencia en los alumnos.

Estas propuestas se están llevando a cabo en la actualidad. Los resultados de su aplicación se trabajarán a finales del curso 2014/15 con el fin de presentar las principales conclusiones obtenidas.

\section{Conclusiones}

La percepción actual de la Didáctica de las Ciencias Experimentales, como resultado del estudio realizado sobre alumnos de 20 curso del Grado en Maestro/a de Educación Primaria, refleja una baja implicación del alumnado por realizar estudios que contengan asignaturas relacionadas con la Ciencia. Resultado de ello es que los alumnos encuestados no tienen perfil científico pues han decidido no estudiar Ciencias, como mínimo, desde finales de la Educación Secundaria Obligatoria. De esta forma resulta poco probable su futura implicación en Ciencia y su promoción en la escuela. A pesar de esta situación, los alumnos muestran un interés creciente por la Ciencia y su didáctica tras cursar asignaturas de Didáctica de las Ciencias Experimentales pero aún así, alrededor de un $30 \%$ del alumnado encuestado no se siente capacitado para enseñar Ciencias en la escuela. Es necesario un esfuerzo para cambiar esta dinámica y para ello se plantean una serie de propuestas con el fín de mejorar el interés por promocionar la Ciencia desde los estudios universitarios hasta la escuela. 
Camina Durántez, A. y Salvador Pérez, M.I. (2007). Condicionantes y Características de los Estudiantes que Inician Magisterio. Estudio Descriptivo y Comparativo entre Especialidades Tendencias Pedagógicas, 12:257-262.

CAÑAL, P. (2007). La investigación escolar, hoy. Alambique Didáctica de las Ciencias Experimentales, 52:9-19.

Cortés Gracia, A.L., y otros (eds.) (2012). Expectativas, necesidades y oportunidades de los maestros en formación ante la enseñanza de las ciencias en la Educación Primaria. Enseñanza de las Ciencias, 30:155-176.

Department of Education and Science (1978). Primary education in England. A survey by HM Inspectors of Schools. Londres: Her Majesty's Stationery Office.

FECYT (2013): Fundación Española para la Ciencia y la Tecnología, FECYT, 2013. Percepción social de la Ciencia y Tecnología 2012. Madrid. http://www.fecyt.es.

FECYT (2012): VI Encuesta de Percepción Social de la Ciencia 2012. EPSCYT 2012. Madrid. http://www.fecyt.es.

Hernández Armenteros, J. (2010): La Universidad española en cifras. Madrid: CRUE (Conferencia de Rectores de las Universidades Españolas).http://www.crue.org/export/sites/Crue/Publicaciones/UEC20 10VOLI.pdf.

Pujol, R. M. (2008). Pensar en la escuela primaria para pensar en la formación de su profesorado, desde la DCE, en el marco del nuevo grado. En M. R. Jiménez Liso (ed.). Ciencias para el mundo contemporáneo y formación del profesorado en Didáctica de las Ciencias Experimentales. Almería: Ed. Universidad de Almería, 354-361.

SCHREINER, C. Y SJøBERG, S. (2004): "Sowing the Seeds of ROSE. Background, Rationale, Questionnaire Development and Data Collection for ROSE (The Relevance of Science Education)". Acta Didactica, 4. Oslo: University of Oslo.

SIøBERG, S. (2005): "Young people and science: Attitudes, values and priorities. Evidence from the ROSE Project". Presentado en EU's Science and Society Forum, Increasing Human Resources for Science and Technology in Europe, 2005. Bruselas.

Siøberg S., Schreiner C. (2010). The Rose Project. Overview and Key Findings. (Oslo, Norway: Univ. of Oslo).

VÁzquez, A. y MANASSERo, M. A. (2008): El declive de las actitudes hacia la ciencia de los estudiantes: un indicador inquietante para la educación científica. Revista Eureka sobre Enseñanza y Divulgación de las Ciencias, 5: 274-292. 
Vázquez-Alonso, Á., Manassero-Mas, M.-A., AceVedo Diaz, J.-A. (2006): An analysis of complex multiple-choice science-technology-society items: Methodological development and preliminary results. Science Education, 90:681-706.

\title{
VIII. Anexo I
}

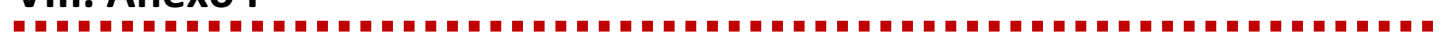

Cuestionario sobre la percepción actual de los alumnos sobre las Ciencias y su Didáctica

\section{Didáctica de las Ciencias Experimentales}

\author{
Promoción de la Ciencia en la Escuela \\ Cuestionario anónimo
}

Edad.

Lugar de nacimiento.

№veces matriculad@ en la asignatura

1. ¿En qué localidad has cursado el Bachillerato o Formación Profesional?

2. ¿Has hecho la Selectividad? SI/NO

¿Qué nota has obtenido sobre 10 ?

3. ¿Fue el Grado en Maestr@ de Educación Primaria tu primera opción? SI/NO

4. ¿Por qué motivo has decidido estudiar esta carrera?

5. ¿Cuántos años hace que estudiaste alguna asignatura de Ciencias?.

6. ¿Qué interés tenía por la Didáctica en Ciencias al inicio de esta asignatura? Pon nota de 0 a 10 , siendo 0 la mínima y 10 la máxima.

7. ¿Qué interés tienes por la Didáctica en Ciencias al finalizar esta asignatura? Pon nota de 0 a 10 , siendo 0 la mínima y 10 la máxima

8. ¿Fomentarías el conocimiento e interés científico cuando seas maestro/educador? $\mathrm{SI} / \mathrm{NO}$

9. ¿Te sientes capacitado para fomentar la Ciencia en los escolares? SI/NO.

10. Introduce cualquier comentario anónimo sobre cómo incentivar el interés por la Ciencia y su didáctica en los futuros maestros: 